\title{
Review on OwnCloud Features for Private Cloud Data Center
}

\author{
Irfan Syamsuddin ${ }^{1}$ Anton Satria Prabuwono ${ }^{2,3}$, \\ Ahmad Hoirul Basori ${ }^{2}$, Arda Yunianta ${ }^{2,4}$ \\ ${ }^{1}$ Department of Computer and Networking Engineering, Politeknik Negeri Ujung Pandang, \\ Makassar, Indonesia \\ ${ }^{2}$ Faculty of Computing and Information Technology in Rabigh, King Abdulaziz University, \\ Jeddah, Saudi Arabia \\ ${ }^{3}$ Faculty of Information Technology, Universitas Budi Luhur, Jakarta, Indonesia \\ ${ }^{4}$ Faculty of Engineering, Mulawarman University,Samarinda, Indonesia
}

\begin{abstract}
It is imperative for government to constantly improve e-government services through innovative technologies in order to cope with tremendous demands coming from citizen side. Although it is realized that e-services to citizens have to be improved and enriched, the realization is not an easy effort since additional budget allocation is usually considered as a hard decision from economic point of view. Cloud computing offers a sound answer to the issue by offering low cost of infrastructure spending, simplifies e-government development and on demand solution to any requirement from user. Practical implementation of OwnCloud for private data center is discussed. Then, the paper briefly reviews several features of OwnCloud that have been modified according to the need of local government and put required assessment on how to implement them appropriately.
\end{abstract}

Keywords - Cloud Computing, Private Cloud, Data Center, OwnCloud, Open Source.

DOI: $10.18421 /$ TEM102-59

https://doi.org/10.18421/TEM102-59

Corresponding author: Irfan Syamsuddin,

Center for Applied ICT Research, Department of Computer and Networking Engineering, Politeknik Negeri Ujung Pandang, Makassar, Indonesia.

Email: irfans@poliupg.ac.id

Received: 13 September 2020.

Revised: 03 May 2021.

Accepted: 10 May 2021.

Published: 27 May 2021.

(c) BY-NC-ND (C) 2021 Irfan Syamsuddin et al; published by UIKTEN. This work is licensed under the Creative Commons Attribution-NonCommercial-NoDerivs 4.0 License.

The article is published with Open Access at www.temjournal.com

\section{Introduction}

It is imperative for government to constantly improve e-government services through innovative improvement in order to cope with tremendous demands coming from citizen side. Although it is realized that e-services to citizens have to be extended, this is not an easy decision since additional budget allocation has to be given for e-government infrastructure enhancement. Government seeks an economically and friendly solution to compromise between needs, e-government infrastructure development and minimum budget allocation [1].

Cloud computing adoptions in e-government are found in many countries nowadays, due to several reasons. With cloud computing e-government might be operated fully centralize which in turn will reduce cost of network management, and make administrator even simpler than before. In fact the most important factor to adopt cloud computing by government sector is economic point of view since using cloud technology will significantly cut the cost of network development and management in comparison to current client server model.

Cloud computing has received lots of attentions by academicians and IT professionals as it has been discussed, deployed, tested and improved. Many technical approaches to enhance cloud's efficiency and service quality are introduced in different ways which have always created innovative ways in this area of study [2].

Similarly, cloud computing has received many business model approached above its implementation both in laboratory test beds as well as in real implementation which makes it economically beneficial. All of these different approaches share the same objectives that are to obtain the most optimum benefit from business perspectives [2].

This paper is organized as follows. In section 2, fundamental concept of cloud computing and open 
source cloud storage is presented. Section 3 presents decision analysis based on fuzzy AHP. Then, section 4 describes in details the development stages of private cloud data center for e-government is presented. Finally, section 5 presents the summary of the study.

\section{Literature Review}

In this section, basic concept of cloud computing technology is presented and followed by description of cloud data storage from open source perspectives.

Cloud computing is unique in terms of four characteristics. The first one is called on-demand service. When users need more computing resources such as CPU, storage or network, they will immediately obtain it with no limitation or restriction cloud from service provider [3].

Secondly, called resource pooling which means cloud services are offered in combination of various resources, but this should be able to serve many clients with different requirements in a simple way [3], [4].

Third characteristic is known as rapid elasticity. From this point of view, clients would be able to enjoy dramatic change of services they need whether scale up when more services or resources are needed or scaled down when some services or resources are not required anymore. This character makes cloud computing is better off than traditional client server mechanisms [4].

The last one is measured service. Cloud computing applies metering capabilities so cloud providers are able to measure how many services or resources have been used by particular users or shared by multiple ones [5].

In terms of cloud service model, there are three basic model on how cloud computing can be applied. The first model is Software as a Service (SaaS). SaaS enable any applications to be hosted and run over the cloud, whenever users need it they able to access the application and work on it on the cloud [4].

The second model of cloud computing is called Platform as a Service (PaaS). Using PaaS model, any clients could use different cloud platform to create any applications and at the same time hosting it on cloud [3]

Finally, Infrastructure as a Service or IaaS model provides any kind of computing infrastructures to rent by users. Instead of having such expensive computing infrastructures which sometimes are not used all the time, cloud computing with IaaS model offers an economic solutions for clients to use extra hardware such as storage, networks, and computing resources as they need and simply release when whenever they do not need [3], [5].
Cloud computing is also recognized in terms of its deployment model. Basically, there are three types of cloud computing model, Public, Private and Hybrid. Public cloud is the deployment of cloud computing by cloud provider to sell their services or resources to cloud clients. Client needs only to rent the required services or resources to cloud provider [4].

In contrary, private cloud is when a client develops own services in house. This approach is slightly similar to client server approach instead it utilized virtualization to operate and share resources internally [4].

Finally, Hybrid cloud is actually when users combine Private and Public clouds according to their needs [3], [5].

Cloud storage is a combination of IaaS and SaaS. It might be seen as IaaS since cloud storage provides a cloud based infrastructure to enable users storing their data on the cloud, while it also can serve as SaaS as it relies on specific software to enable data storing mechanisms on the cloud [6].

Cloud storage tries to replace common media storage by providing similar storage. Cloud storage provides media for saving, editing, and managing data on the cloud scalable as needed by the users [7].

Currently, cloud storage is available both in open and closed source forms. In this study, only open sources one are discussed as this is the main topic to be described. Four open source cloud storage solutions mentioned below are considered as potential candidates for cloud adoption in egovernment data center creation

\section{- OwnCloud}

OwnCloud is an open source "Dropbox like" cloud storage written in PHP, JavaScript and available for Windows, Linux, OS X desktops. OwnCloud claims several advantages such as easy installation, GUI based user and storage management, multi documents supports, data sharing with encryption mode, as well as synchronization with different clients gadgets [8]. It is available in $\mathrm{iOS}$ and Android platforms.

\section{- SeaFile}

Seafile is another cloud solution for storage written in $\mathrm{C}$ and Python. It is available in premium and open source for Windows, Linux, and OS X. Seafile also support mobile client in $\mathrm{iOS}$, and Android. SeaFile in open source version offers several features such as file editing on the cloud, synchronization with clientside encryption. In addition for paid version, more features are provided such as user logging and text search [9].

\section{- Cozy}

Cozy is another open source cloud storage written in Node.JS. It can either be used as private personal storage or trust Cozy faderated cloud servers. It is available for all platforms both desktop and mobile 
[10]. Main features of Cozy include ability to store different kind of client's data in on cloud while synchronizing them at the same time. In addition, it also supports GIT url for sharing applications and HTML5 for dynamic requirements.

\section{- Syncany}

Syncany is known by its small size among aforementioned open source storage. However, it offers many features like other cloud software such as various types of data support, simple sync process with command line. The advantage of Syncany is data encryption feature using 128-bit AES+Twofish/GCM [11].

\section{Methodology}

The study applies Fuzzy Analytic Hierarchy Process (AHP) which is basically based on AHP in combination with Fuzzy Set Theory. AHP is based on multi-criteria decision technique introduced by Saaty (1980) to solve problems involving different point of views, various criteria which sometimes are conflicting each other, with a number of alternatives to be chosen or selected (Chang, 1992).

AHP is considered as useful to deal with problems involving multiple criteria, in qualitative and quantitative approaches (Markaki, Charilas, \& Askounis, 2010). The AHP technique is developed through six key stages:

a. Define the goal to be solved.

b. Construct a hierarchical structure with elements of decision. In simplest one, it consists of three layers, goal, criteria and alternatives.

c. Pair comparisons at all layers between criteria and between alternatives.

d. Calculate the consistency level to ensure judgments are consistent.

e. Calculate the relative weights of the decision elements.

f. Rank the final weight in descending way.

These are the steps incorporated in standard AHP in combination with Fuzzy Set Theory. Fuzzy version of Analytic Hierarchy Process is applied as multi criteria decision analysis [12], [14]. Fuzzy set theory is oriented to the rationality of uncertainty due to imprecision or vagueness [12]. In the field of Multi Criteria Decision Making, fuzzy set theory has given a significant contribution by accepting uncertainty and inconsistent judgment as a nature of human decision making [12].

Fuzzy set is determined by a membership function whose membership is defined by Lower, Medium and Upper numbers. The membership function of $\mathrm{M}=(1, \mathrm{~m}, \mathrm{u})$ is given by

$$
\mu(x)=\left\{\begin{array}{l}
\frac{x-l}{m-l} \quad \text { if } l \leq x \leq m \\
\frac{u-x}{u-m} \quad \text { if } m \leq x \leq u \\
0 \quad \text { if } \quad x<l \text { or } x>u
\end{array}\right.
$$

Based on this function, it can be represented in graphic of fuzzy number as seen in Figure 1. These fuzzy numbers are applied to Analytic Hierarchy Process [17], to establish the decision analysis.

These fuzzy numbers may be determined into ways, whether by using directly according to the decision maker understanding or they can be derived from linguistic variables in a verbal scale.

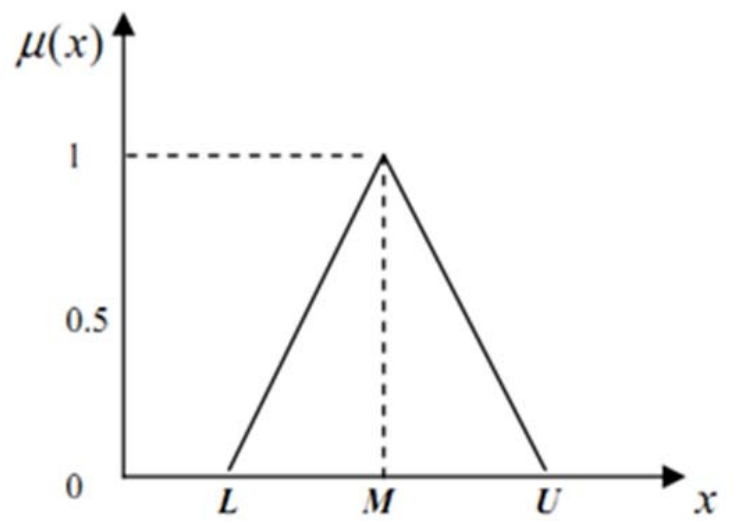

Figure 1. Triangular fuzzy numbers

\section{Analysis and Discussion}

Inspired by Wang's cloud adoption model [13], a novel cloud adoption framework for open source cloud storage decision making is proposed. It has four main aspects to consider in making decision, namely response time, accuracy stability and security.

The framework is basically structured according to the Analytic Hierarchy Process to develop required hierarchy for decision analysis. However, the decision analysis employs Fuzzy approach using fuzzy triangular numbers as means of linguistic variables [14].

In other words, instead of using crisp number of classical AHP this approach utilizes fuzzy numbers to accommodate fuzziness that commonly found in technical decision making such as cloud computing [14].

The framework consists of three layers namely, goal, criteria and alternatives. At the top level, the goal is defined as selecting the most appropriate open source cloud storage for being adopted in egovernment data center project.

Then, there are four criteria at the second layer, namely Response Time, Accuracy, Stability and Security. Finally, the alternatives are the four candidates of open source cloud storage. 
Experts who are responsible to make the decision in this case were involved to make judgements among four candidates. After all steps of pairwise comparisons have been completed, consistency ratio calculation was below 0.1 [12].

The first place goes to OwnCloud with 0.286, while the second one is given to SeaFile which accounted for 0.247 , followed by Syncany and Cozy which accounted for 0.235 and 0.232 respectively.

The finding clearly suggests that among all candidates, OwnCloud is the best open source cloud storage to be adopted by e-government considering four aspects mentioned previously, response time, accuracy, stability, and security.

After selecting the best open source cloud storage, the next stage is establishing the private cloud data center. In terms of software license cost, since it runs above Linux machine, the cloud data center is economically and friendly with no licence required. Therefore, a number of OwnCloud implementations reported in several government institutions as discussed in [15] and [19] while few of them were reported in business [19].

OwnCloud also reported has been deployed in several university [16] and research institutions [17] developed private data center. Similarly, OwnCloud has also attracted the interest of health experts to adopt it in their network [18] with a number of modifications [20].

As suggested in [16] and [19], due to its simplicity and various features as well as "Dropbox like" functionalities, these have made OwnCloud very interactive to be tested and deployed. Whole processes are not complicated to instal, and preparation is done until establishing working private cloud storage [19].

The development stage begins with preparing the Linux machine with approximately $16 \mathrm{~GB}$ of RAM for server. Apache server and MySQL database has to be installed and tested in order to ensure that both are running well.

Once the package of OwnCloud has been downloaded from the official website [8], installation can be performed. It takes some minutes from setting up to run OwnCloud data storage in Linux server.

The next step after fresh installation is to change default main administrator details such as username and password. This part is very critical for OwnCloud based data center systems in order to ensure confidentiality of the whole systems and preventing data from unauthorized parties in the future.

It is important to note that OwnCloud offers easy editing and modification [21]. Standard homepage has undergone significant modification to personalize the private data center look. Figure 2 shows a new look of e-Gov Cloud Data Center with government logo for personalization.

Along with homepage modification is user's administration. In this case, the systems will not allow unverified user to fulfill registration without accepted credentials from the system administrator. OwnCloud provides such assessment by giving all rights for user registration to system administrator only. Every new user has to ask username the administrator by giving required information, and if accepted then the administrator will assign username and password for the user.

Although this approach is good for security reason, it is found that such strict registration procedure is somewhat inefficient and time consuming. This is due to all registration should be performed through one gate called "administrator gate" and new user should wait for some time before permission granted by administrator. This will become serious efficiency issues for large number of government employee.

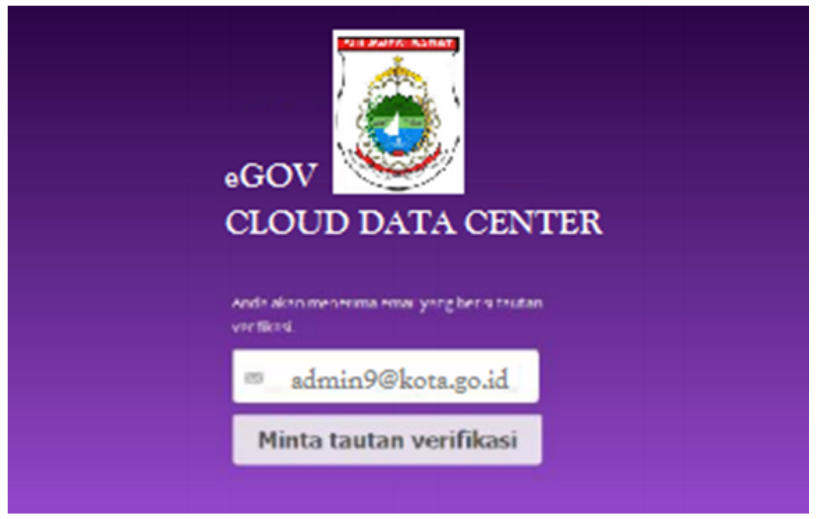

Figure 2. User Authentication

To deal with such inefficiency in user registration, a new email based verification system for new user registration is applied to the system. This approach significantly eases the task of administrator by enabling new user to make registration and waiting for verification link via email.

In this case, only users with authorized email with domain name of "kota.go.id" will be acceptable. Once new user registration is supplied with different email domain, and then automatically will not be accepted by the new systems for registration. This rejection will be sent to email and also appears on the homepage of the private cloud data center.

The next feature called user and group have been tested and applied by following current government departments. It is customized properly to adopt the requirement of the municipality before applying it in real environment.

There are ten groups are created by main administrator such as Dinas Kesehatan, Dinas Pekerjaan Umum, Dinas Pendidikan and others which are actual government department names (Figure 3). Once all groups exist, all employees with 
their usernames are associated with specific group according to their current position at the department.

Within each group, a group administrator is selected and set up by main administrator by giving specific roles such as managing user within the group, adding document, sharing document, and deleting document. Such decentralized management in private data center is useful to reduce administration complexity at main administrator side, while reduce processing time by allowing several processes to be done specifically at group level.

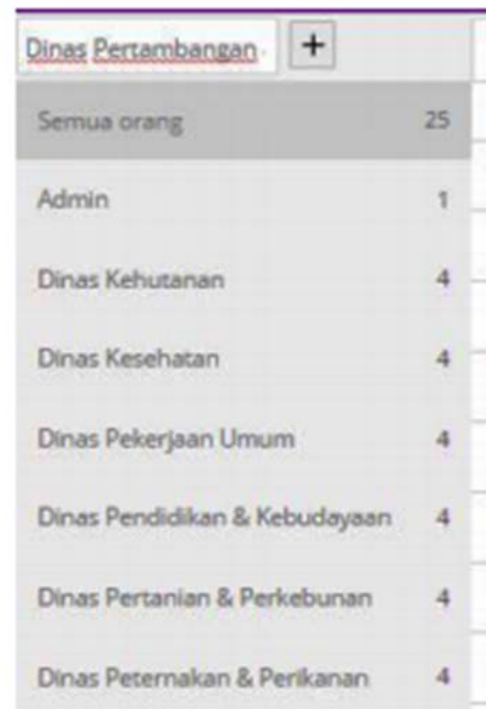

Figure 3. Group of government departments

In terms of types of document to store, group administrator let all group members to store any kind of document.

The supported text files for file sharing are pdf, doc, rtf, xls and txt many others as seen in Figure 4. Picture files are also allowed, while administrator strictly prevents any application files such as exe, vbs and others to be uploaded to the private data center due to security reason. All document files will be automatically sharable within the same group, but it requires group administrator permission to share it with users from different group.

$$
\begin{aligned}
& <\text { Bagi tautan } \\
& 1 \text { Data PraSejahtera TW01 2018.pdf } 2.1 \mathrm{MB}
\end{aligned}
$$

Figure 4. File sharing

In case of public information, which is regarded as information that has to be accessible freely to citizens such as government announcement, there is no need to ask sharing permission. The private cloud data center system will automatically assign specific embedded link that can be put directly in government website.

From the perspective of mobility, OwnCloud offers client version in $\mathrm{iOS}$ and Android which can be modified according to the main private data center modification. Mobile client is excellent tool for mobile employees who need to keep accessing government private data center from any mobile devices.

Despite accessing their account directly from the website of private data center, government employee can work from distance and synchronize the data from different endpoint to private data center. Data synchronization can simply be processed only by clicking "Connect" button in mobile client version as depicted in Figure 5. Time for synchronization process depends upon size of data and network conditions.
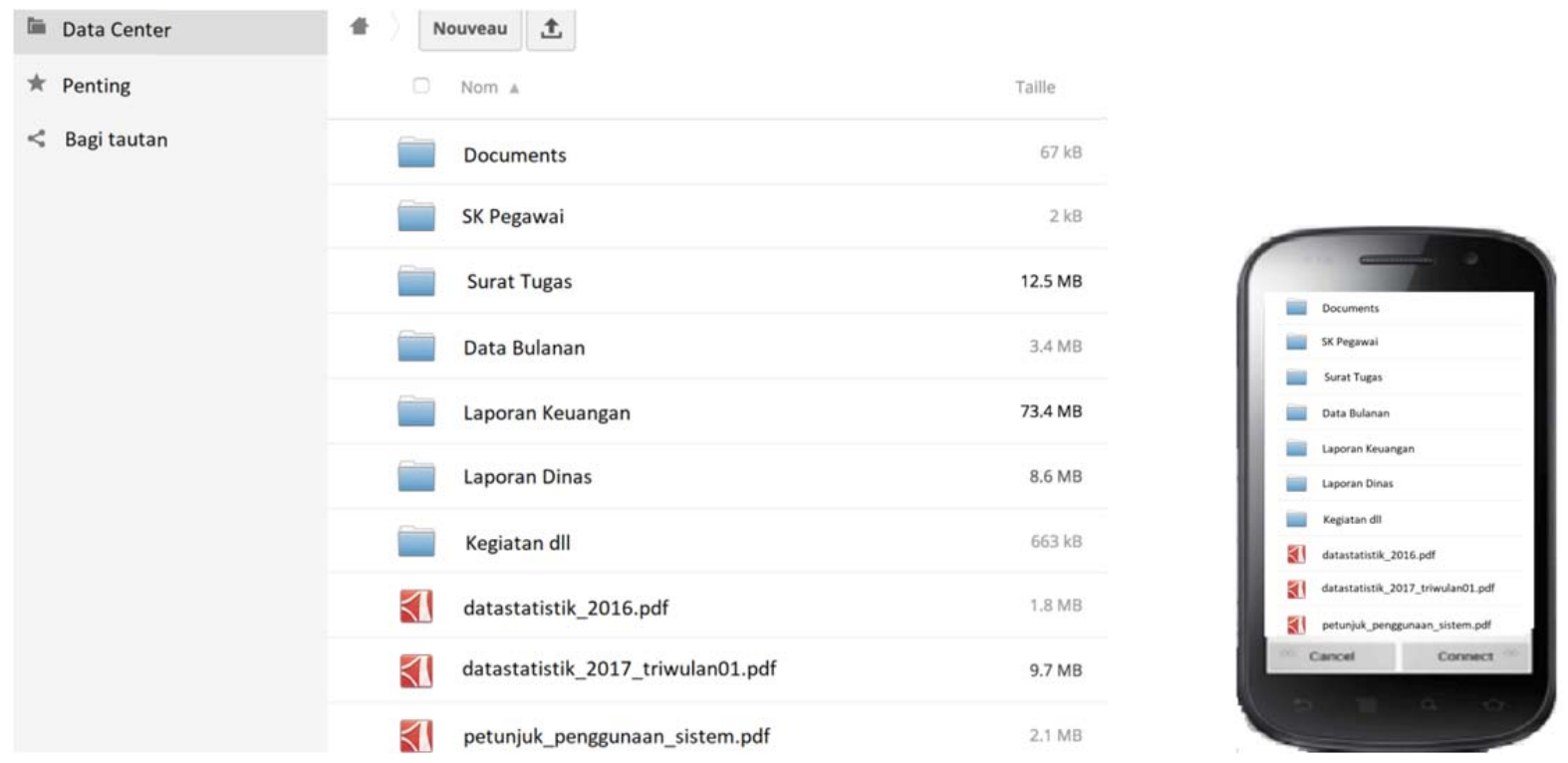

Figure 5. Synchronization between cloud data center and mobile client 
Table 1. Summary of OwnCloud features in Private Cloud Data Center

\begin{tabular}{|c|c|c|c|c|}
\hline No & Feature & Description & Mode & Status \\
\hline 1 & Main Administrator & $\begin{array}{l}\text { Feature for super administrator to } \\
\text { manage all users and functions }\end{array}$ & Standard & Working \\
\hline 2 & Group Administrator & $\begin{array}{l}\text { Feature for all group administrators to } \\
\text { specific users and their associated } \\
\text { groups }\end{array}$ & Modified & Working \\
\hline 3 & Group Management & $\begin{array}{l}\text { Feature to manage group and its scope } \\
\text { of ability to handle user and document }\end{array}$ & Modified & Working \\
\hline 4 & User Profile & $\begin{array}{l}\text { Feature for user to describe required } \\
\text { profile }\end{array}$ & Standard & Working \\
\hline 5 & Email & Feature for user to maintain email & Modified & Working \\
\hline 6 & Document Type & $\begin{array}{l}\text { Feature for determining types of } \\
\text { document approved in the private cloud } \\
\text { data center }\end{array}$ & Standard & Working \\
\hline 7 & File Sharing & $\begin{array}{l}\text { Feature for particular users to share } \\
\text { documents stored in the private data } \\
\text { center }\end{array}$ & Modified & Working \\
\hline 8 & Mobile Client & $\begin{array}{l}\text { Feature to enable user interface in } \\
\text { mobile, tablet or smartphone }\end{array}$ & Standard & Working \\
\hline 9 & Synchronization & $\begin{array}{l}\text { Feature to enable user to synchronize } \\
\text { document from any endpoint }\end{array}$ & Modified & Working \\
\hline 10 & $\log$ & $\begin{array}{l}\text { Feature to activate history of all } \\
\text { activities within the private cloud data } \\
\text { center }\end{array}$ & Modified & Working \\
\hline
\end{tabular}

For this purpose, client side application of OwnCloud was tested from different endpoints such as tablet, and smartphone (iOS and Android). The result is excellent as they can synchronize data appropriately. At this stage, all required functions of e-government private data center operate perfectly.

The last feature exploited in this study is logging. All user activities as well as any documents are well monitored by main administrator via this feature. Such log history is important to deal with any misconduct by internal users or by cyber attackers from outside the data center systems.

In short, all features of OwnCloud used in establishing e-government private cloud data center provider are working perfectly both in standard and modified modes. There are ten features of OwnCloud utilized in this case of establishing private cloud data center for e-government. Table 1 summarizes the features and explains how individual feature is applied into the private cloud data center.

In the near future, the study will be tested in large number of users accessing the system simultaneously in order to evaluate the quality of service (QoS) of the e-government private data center. Besides, future research might be directed to the improvement of mobile cloud security by following recommendation proposed by Do,et.al. [22] in terms of modifying Android File system Permission (AFP), or conducting forensics analysis of mobile cloud as suggested in [23] or enhancing its security by various authentication protocols in combination with Android Apps [24] or RFID technology [25].

\section{Summary}

This study briefly describes how OwnCloud, an open source cloud data storage has been applied in developing a private data center. The solution has proven to balance proportion between urgent need of new data storage in one hand and limited budget constraints on the other hand.

The study was carried out within two steps. Firstly, a candidacy stage by selecting several options of open source cloud storages. Secondly, deployment stage which describes steps of establishing private cloud data center for local government needs.

Finally, brief review on ten main features of OwnCloud is presented; in which six of them were modified according to the requirements while four others left in standard format. The uniqueness of this study in lies in the usability of Owncloud in developing cloud data center for government would pave future research in different ways of implementations of cloud computer data center in the future. 


\section{References}

[1]. Vaquero, L. M., Rodero-Merino, L., Caceres, J. \& Lindner, M., (2008) A break in the clouds: towards a cloud definition. ACM SIGCOMM Computer Communication Review, 39, 1, 50-55.

[2]. Lu, G., \& Zeng, W. H. (2014). Cloud computing survey. In Applied Mechanics and Materials (Vol. 530, pp. 650-661). Trans Tech Publications Ltd.

[3]. Bilgaiyan, S., Sagnika, S., \& Sahu, S. S. (2014). Cloud computing: Concept, terminologies, issues, recent technologies. Research Journal of Applied Sciences, 9(9), 614-618.

[4]. Buyya, R., Yeo, C. S., Venugopal, S., Broberg, J., \& Brandic, I. (2009). Cloud computing and emerging IT platforms: Vision, hype, and reality for delivering computing as the 5th utility. Future Generation computer systems, 25(6), 599-616.

[5]. Rajkumar, M.N., Palaniswami, S., Kumar, V. \& Vijayabhasker, R. (2014), An Analysis on the Characteristics of Cloud Computing. International Journal of Soft Computing, 9,1, 10-19.

[6]. Stefanov, E., \& Shi, E. (2013, May). Oblivistore: High performance oblivious cloud storage. In 2013 IEEE Symposium on Security and Privacy (pp. 253267). IEEE.

[7]. Yang, H. L., \& Lin, S. L. (2015). User continuance intention to use cloud storage service. Computers in Human Behavior, 52, 219-232.

[8]. Patawari, A. (2013). Getting started with ownCloud. Packt Publishing Ltd.

[9]. Yang, S., Jiang, L., Zhu, S., \& Dai, L. (2013, November). Research and application of private cloud storage platform in high schools based on seafile. In 2013 6th International Conference on Intelligent Networks and Intelligent Systems (ICINIS) (pp. 2528). IEEE.

[10]. Tran-Van, P., Anciaux, N., \& Pucheral, P. (2017, November). SWYSWYK: a new Sharing Paradigm for the Personal Cloud. In International Conference on Advanced Data Mining and Applications (pp. 839845). Springer, Cham.

[11]. Teing, Y. Y., Dehghantanha, A., Choo, K. K. R., Muda, Z., \& Abdullah, M. T. (2017). Greening cloudenabled big data storage forensics: Syncany as a case study. IEEE Transactions on Sustainable Computing, 4(2), 204-216.

[12]. Kahraman, C., \& Karaşan, A. (2018). A Literature Survey on the Usage of Fuzzy MCDM Methods for Digital Marketing. Intelligent Systems: Concepts, Methodologies, Tools, and Applications, 54-72.

[13]. Wang, P., Gao, R. X., \& Fan, Z. (2015). Cloud computing for cloud manufacturing: benefits and limitations. Journal of Manufacturing Science and Engineering, 137(4).
[14]. Syamsuddin, I., \& Hwang, J. (2010, October). A new fuzzy MCDM framework to evaluate e-government security strategy. In 2010 4th International Conference on Application of Information and Communication Technologies (pp. 1-5). IEEE.

[15]. Chang, Y. S., Lee, Y. K., Juang, T. Y., \& Yen, J. S. (2013). Cost evaluation on building and operating cloud platform. International Journal of Grid and High Performance Computing (IJGHPC), 5(2), 43-53.

[16]. Mościcki, J. T., \& Lamanna, M. (2014, June). Prototyping a file sharing and synchronization service with Owncloud. In Journal of Physics: Conference Series (Vol. 513, No. 4, p. 042034). IOP Publishing.

[17]. Hildmann, T., \& Kao, O. (2014, June). Deploying and extending on-premise cloud storage based on ownCloud. In 2014 IEEE 34th International Conference on Distributed Computing Systems Workshops (ICDCSW) (pp. 76-81). IEEE.

[18]. Divya, T., \& Shanmugapriya, L. (2016, January). Two-level security in shared $\mathrm{m}$-healthcare system using owncloud. In 2016 International Conference on Computing Technologies and Intelligent Data Engineering (ICCTIDE'16) (pp. 1-6). IEEE.

[19]. Charif, B., \& Awad, A. I. (2014, September). Business and government organizations' adoption of cloud computing. In International Conference on Intelligent Data Engineering and Automated Learning (pp. 492-501). Springer, Cham.

[20]. Hani, A. F. M., Paputungan, I. V., Hassan, M. F., Asirvadam, V. S., \& Daharus, M. (2014, June). Development of private cloud storage for medical image research data. In 2014 International Conference on Computer and Information Sciences (ICCOINS) (pp. 1-6). IEEE.

[21]. Saha, A. (2012). A look at ownCloud. Linux Journal, 2012(218), 1.

[22]. Do, Q., Martini, B., \& Choo, K. K. R. (2014, September). Enforcing file system permissions on android external storage: Android file system permissions (afp) prototype and owncloud. In 2014 IEEE 13th International Conference on Trust, Security and Privacy in Computing and Communications (pp. 949-954). IEEE.

[23]. Daryabar, F., Dehghantanha, A., Eterovic-Soric, B., \& Choo, K. K. R. (2016). Forensic investigation of OneDrive, Box, GoogleDrive and Dropbox applications on Android and iOS devices. Australian Journal of Forensic Sciences, 48(6), 615-642.

[24]. Syamsuddin,I., Ihdianty, S., Tungadi,E., Kasim,K., \& Irawan, I. (2020) XTEA Cryptography Implementation in Android Chatting App, Journal of Information Technology and Its Utilization, 3,2, 3643.

[25]. Alaba, F. A., Othman, M., Hashem, I. A. T., \& Alotaibi, F. (2017). Internet of Things security: A survey. Journal of Network and Computer Applications, 88, 10-28. 